\title{
Genetic variation in heat tolerance-related traits in a population of wheat multiple synthetic derivatives
}

\author{
Awad A. E. Elbashir ${ }^{1,3)}$, Yasir S. A. Gorafi, ${ }^{2,3)}$, Izzat S. A. Tahir ${ }^{3)}$, Ashraf. M. A. Elhashimi ${ }^{3)}$, \\ Modather G. A. Abdalla ${ }^{3)}$ and Hisashi Tsujimoto ${ }^{* 2)}$ \\ 1) United Graduate School of Agricultural Sciences, Tottori University, Tottori 680-8553, Japan \\ 2) Arid Land Research Center, Tottori University, 1390 Hamasaka, Tottori 680-0001, Japan \\ 3) Agricultural Research Corporation, PO Box 126, Wad Medani, Sudan
}

\begin{abstract}
In wheat (Triticum aestivum L.) high temperature $\left(\geq 30^{\circ} \mathrm{C}\right)$ during grain filling leads to considerable reduction in grain yield. We studied 400 multiple synthetic derivatives (MSD) lines to examine the genetic variability of heat stress-adaptive traits and to identify new sources of heat tolerance to be used in wheat breeding programs. The experiment was arranged in an augmented randomized complete block design in four environments in Sudan. A wide range of genetic variability was found in most of the traits in all environments. For all traits examined, we found MSD lines that showed better performance than their parent 'Norin 61' and two adapted Sudanese cultivars. Using the heat tolerance efficiency, we identified 13 highly heat-tolerant lines and several lines with intermediate heat tolerance and good yield potential. We also identified lines with alleles that can be used to increase wheat yield potential. Our study revealed that the use of the MSD population is an efficient way to explore the genetic variation in Ae. tauschii for wheat breeding and improvement.
\end{abstract}

Key Words: bread wheat, heat stress, drought, germplasm enhancement, wild species.

\section{Introduction}

High temperature is an important constraint to bread wheat productivity in tropical and subtropical environments; it causes morphological and physiological changes at all stages and results in considerable yield losses (Al-khatib and Paulsen 1990, Tahir and Nakata 2005, Tahir et al. 2006, Tewolde et al. 2006). Wheat is adapted to perform well at $18-24^{\circ} \mathrm{C}$. High temperature during flowering decreases grain number, and temperature above $34^{\circ} \mathrm{C}$ after anthesis leads to reduce yield potential (Asseng et al. 2011, Ferris et al. 1998, Lobell et al. 2012). Asseng et al. (2015) reported that an increase of $1{ }^{\circ} \mathrm{C}$ reduces grain yield by $6 \%$. Physiological parameters such as chlorophyll content, canopy temperature, and normalized difference vegetation index are widely used as indicators of wheat heat tolerance (Al-khatib and Paulsen 1990, Amani et al. 1996, Hazratkulova et al. 2012).

Although genetic variation for heat stress tolerance in elite wheat germplasm is limited, considerable genetic variation is present in wild relatives ( $\mathrm{Li}$ et al. 2014). Both Aegilops tauschii $(2 \mathrm{n}=2 \mathrm{x}=14$, DD) and durum wheat

Communicated by Takao Komatsuda

Received May 11, 2017. Accepted August 4, 2017.

First Published Online in J-STAGE on December 1, 2017.

*Corresponding author (e-mail: tsujim@alrc.tottori-u.ac.jp)
(Triticum turgidum subsp. durum, $2 \mathrm{n}=4 \mathrm{x}=28$, AABB) are the ancestors of bread wheat (Triticum aestivum L., $2 n=6 x=42$, AABBDD) and the secondary gene pools for improving the resistance or tolerance of bread wheat to biotic and abiotic stresses. Synthetic hexaploid wheat (SHW) is usually produced by crossing durum wheat and Ae. tauschii to enable the use of these genetic resources. Sharma et al. (2014) found four highly heat-tolerant lines among 24 SHW lines. Furthermore, SHWs are tolerant to high temperatures $\left(35-40^{\circ} \mathrm{C}\right)$ during the grain filling stage (Van Ginkel and Ogbonnaya 2007).

A synthetic wheat derivative from the International Maize and Wheat Improvement Center (CIMMYT) with early maturity, a short grain filling period, and good grain quality was found to be suitable for late planting in southern Spain and to produce a higher yield than commercial varieties. In 2003, this derivative was registered as a new cultivar, 'Carmona' (Kazi and Van Gikel 2004). Another new SHW line, 'Super wheat', developed at the National Institute of Agricultural Botany, Cambridge University, could increase wheat productivity up to $30 \%$ (http://www.niab. com/news_and events/article/281).

Despite these developments, only a limited number of Ae. tauschii lines, and therefore only a small portion of the high diversity of this species, are used in breeding programs. For the breeding program to overcome this problem and to 
ensure proper and extensive utilization of this tremendous diversity, a multiple synthetic derivatives (MSD) has been developed (Gorafi et al. unpublished, Tsujimoto et al. 2015). In 2013, we grew 1000 plants from the MSD population under heat stress in Sudan, out of which six plants were selected visually as heat tolerant, and two of them were then confirmed to be heat tolerant (Elbashir et al. 2017).

In this study, we randomly selected $400 \mathrm{BC}_{1} \mathrm{~F}_{4}$ plants from the MSD population and evaluated them in four environments in Sudan, to identify new sources for the development of heat-tolerant high-yielding wheat cultivars.

\section{Materials and Methods}

\section{Plant materials}

The MSD population was developed by crossing and backcrossing the Japanese wheat cultivar 'Norin 61' to 43 SHW lines produced by crossing the durum wheat cultivar 'Langdon' and 43 different Ae. tauschii accessions (Matsuoka and Nasuda 2004). In this study, we used 400 $\mathrm{BC}_{1} \mathrm{~F}_{4}$ lines randomly selected from the MSD population, their backcross parent 'Norin 61' (hereafter N61) as a control to show the effect of the wild genes on the improvement of N61 heat stress adaptability, and the two heat-tolerant Sudanese cultivars 'Goumria' and 'Imam' as checks to evaluate the heat stress adaptability of the MSD lines. The 400 MSD lines were selected in 2014/2015 at the Arid Land Research Center, Tottori University, Japan $\left(35^{\circ} 32^{\prime} \mathrm{N}\right.$, $134^{\circ} 13^{\prime} \mathrm{E}, 6$ masl).

\section{Experimental sites, design and field management}

We evaluated the plants in four agro-ecological environments at three sites in Sudan (Dongola, Hudeiba, and Wad Medani) in 2015/2016. Dongola Research Farm (DON) is located in Northern State $\left(19^{\circ} 08^{\prime} \mathrm{N}, 30^{\circ} 27^{\prime} \mathrm{E}, 239\right.$ masl $)$ and has a high-terrace soil ( $\mathrm{pH} 8$ ) with low organic matter content $(<5 \%)$. The texture is sandy clay loam at $0-30 \mathrm{~cm}$ and silty clay loam at $30-60 \mathrm{~cm}$. Hudeiba Research Farm (HUD) is located in River Nile State $\left(14^{\circ} 40^{\prime} \mathrm{N}, 33^{\circ} 50^{\prime} \mathrm{E}\right.$, 409 masl) and has a middle-terrace soil (Karu; pH 8). Wad Medani (MED) is located in the central clay plain of Gezira State $\left(14^{\circ} 24^{\prime} \mathrm{N}, 2^{\circ} 33^{\prime} \mathrm{E}, 407 \mathrm{masl}\right)$ and has a heavy clay soil $(\mathrm{pH} 8.0-8.4)$ with low organic matter content $(<5 \%)$ and low levels of nitrogen (380 ppm) and phosphorus. We expected the temperatures to be low or optimum for wheat cultivation at DON and high at MED. At all three sites, we used an augmented randomized complete block design with eight blocks, three replicated checks per block and 54 entries per block. At DON and HUD, seeds were sown in the $4^{\text {th }}$ week of November. At MED, seeds were sown in the $4^{\text {th }}$ week of November (optimum sowing; MED/OS) and in the $2^{\text {nd }}$ week of December (late sowing; MED/LS) to ensure plant exposure to high temperatures at the grain filling stage.

Before sowing, seeds were treated with the insecticide Gaucho (imidacloprid, 35\% WP, Bayer Crop Science, USA) at $1 \mathrm{~g} \mathrm{~kg}^{-1}$ seed, mainly to control termites and aphids.
Seeds were sown at $120 \mathrm{~kg} \mathrm{ha}^{-1}$ manually. Each line was planted in a plot of four rows, $0.5 \mathrm{~m}$ long and $0.2 \mathrm{~m}$ apart. Phosphorus was applied as superphosphate by furrow placement before sowing at $18.8 \mathrm{~kg} \mathrm{P} \mathrm{ha}^{-1}$. Nitrogen was splitapplied as urea at the three-leaf stage (second irrigation) and the tillering stage (fourth irrigation) at $86 \mathrm{~kg} \mathrm{~N} \mathrm{ha}^{-1}$. Irrigation was applied every 10-12 days. The fields were handweeded at least twice.

\section{Evaluated traits}

The following morphological and physiological traits were evaluated: days to heading (DH), days to maturity (DM), grain filling duration (GFD), plant height (PH), peduncle length (PL), biomass (BIO), grain yield (GY), harvest index $(\mathrm{HI})$, kernel number per spike $(\mathrm{KS})$, thousandkernel weight (TKW), and grain number per $\mathrm{m}^{2}\left(\mathrm{Gm}^{-2}\right)$. At the grain filling stage, we measured the following physiological parameters: canopy temperature (CT), chlorophyll content (SPAD), and normalized difference vegetation index (NDVI). The measurements were performed according to Pask et al. (2012). The heat tolerance efficiency (HTE) of each line was calculated as HTE $=100 *$ Ysi/Ypi (Fischer and Wood 1981), where Ysi is GY under high temperature and Ypi is GY under low temperature. The first HTE calculation was performed using the GY values at DON (considered a cool environment) and at MED/LS (hot environment); the second HTE calculation was performed using the GY values at MED/OS (cool environment) and MED/LS (hot environment).

\section{Statistical analysis}

Analysis of variance was carried out using Plant Breeding Tools software (PBTools, version 1.4. International Rice Research Institute, Los Baños, The Philippines; http://bbi. irri.org/products) for each environment separately due to the significant environmental effect. The MSD lines that did not head were considered as missing values in the analysis of the data. Simple correlation coefficients between GY and other traits were calculated for all genotypes in each environment. To identify heat-tolerant lines, high-yielding lines, and heat-tolerant high-yielding lines, we performed regression analysis in two ways: first, by using the GY data at DON as the optimum condition and the HTE data calculated using DON vs. MED/LS; second, by using the GY data at MED/OS as the optimum condition and the HTE data calculated using MED/OS and MED/LS. We calculated the heritability by PBTools using the formula of broad sense heritability $\left(h^{2}=\mathrm{VG} / \mathrm{VP}\right)$, where VG is the genotypic variance and VP is the phenotypic variance, considering genotype as a random effect. The heritability was classified as low $(0-$ $0.30)$, moderate $(>0.30-0.60)$, or high $(>0.60)$ (Allard 1960).

\section{Results}

\section{Temperature at three experimental sites}

The weekly maximum and minimum temperatures during 


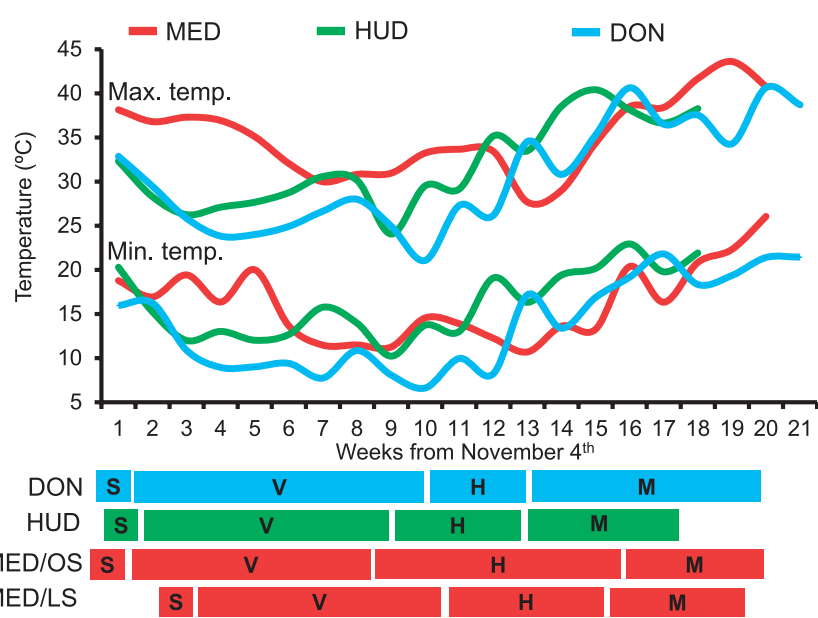

Fig. 1. Weekly maximum and minimum temperatures at Dongola (DON), Hudeiba (HUD), and Gezira (Wad Medani; MED) Research Farms starting from the 4 th of November. OS, optimum sowing; LS, late sowing; $\mathrm{S}$, sowing time; $\mathrm{V}$, vegetative stage; $\mathrm{H}$, heading stage; $\mathrm{M}$, maturity stage.

the cropping season in the four environments are shown in Fig. 1. DON was the coolest environment during sowing, vegetative growth, and heading (up to the $12^{\text {th }}$ week) and after the $16^{\text {th }}$ week (late maturity), whereas MED/OS and MED/LS were the hottest during these time periods. HUD was the hottest and MED/OS and MED/LS were the coolest environments between the $12^{\text {th }}$ week (late heading) and the $16^{\text {th }}$ week (early maturity). Overall, we considered DON and MED/OS as cool environments and HUD and MED/LS as hot environments.

\section{Performance of N61, the genetic background of MSD} lines, under Sudanese conditions

We first investigated the adaptability of N61 in comparison with the two Sudanese cultivars adapted to high temperature. The DH values of N61 were similar to those of the Sudanese cultivars at DON but were significantly lower at HUD and MED/LS (Fig. 2A, Supplemental Table 1). The DM values were similar between N61 and the Sudanese cultivars in all environments (Fig. 2B, Supplemental Table 1). N61 was significantly taller in PH than 'Goumria' at MED/LS, and its PL was similar between all the environments (Fig. 3, Supplemental Table 1). TKW of N61 was significantly lower than that of 'Imam' at HUD (Fig. 5B). All other parameters measured were similar between N61 and the Sudanese cultivars in all environments (Supplemental Table 1). All these data indicate that N61 is adapted to heat stress.

\section{Days to heading and maturity and grain filling duration in MSD lines}

MSD lines showed high variability in DH, DM, and GFD, and $8 \%$ of the 400 lines at DON, $5 \%$ at MED/OS,
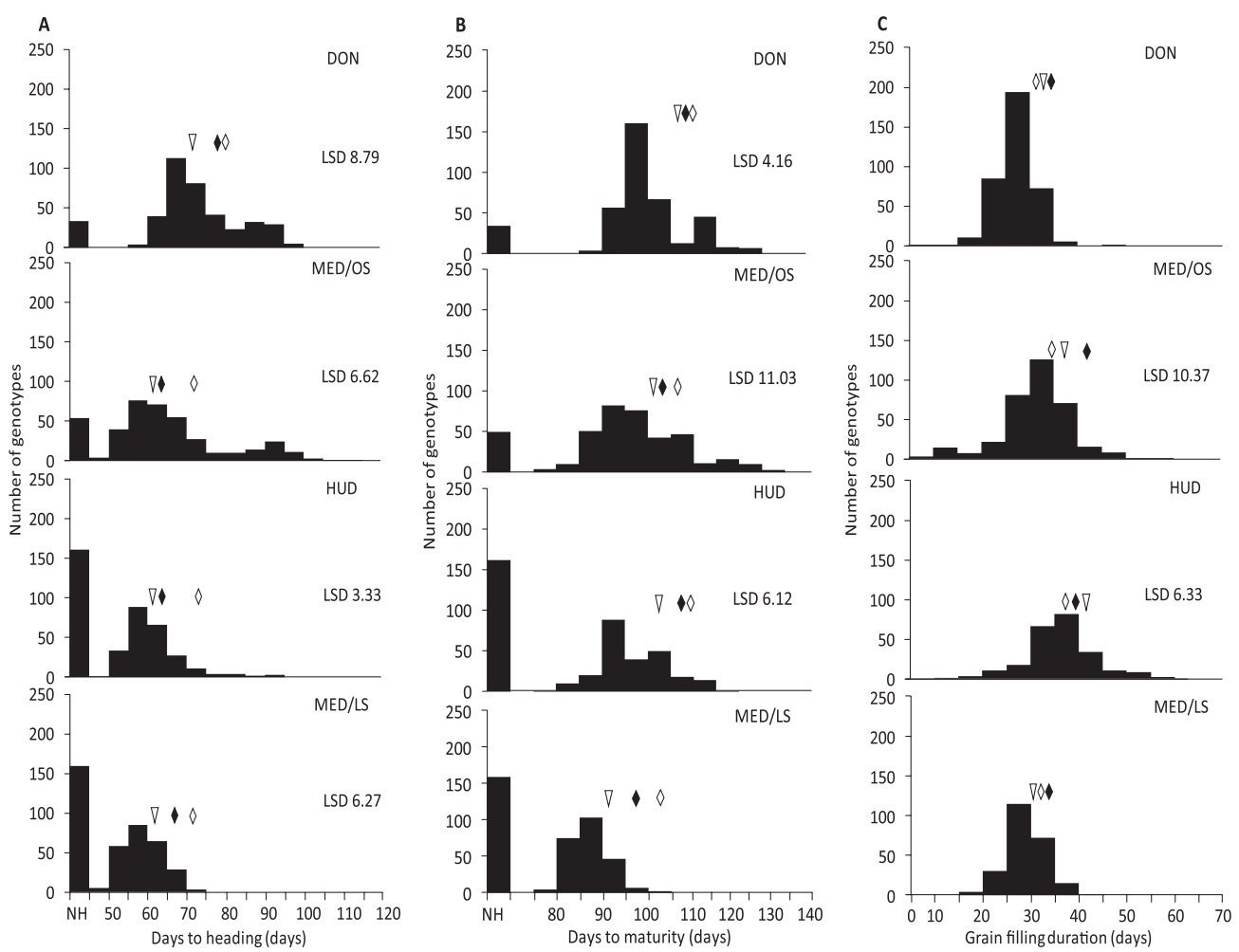

Fig. 2. Frequency distribution of days to heading (A), days to maturity (B) and grain filling duration (C) of the MSD lines, their parent 'Norin 61 ' (triangle) and the adapted Sudanese cultivars 'Goumria' (black rhombus) and 'Imam' (white rhombus) of four environments of Dongola (DON), Wad Medani optimum sowing (MED/OS), Hudieba (HUD) and Wad Medani late sowing (MED/LS) in 2015/16. 

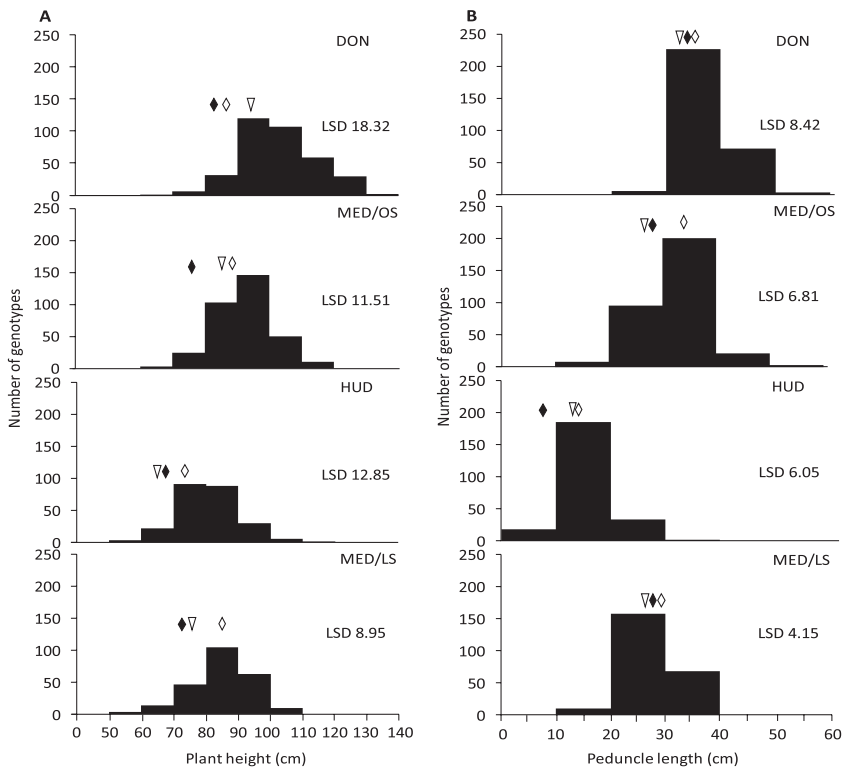

Fig. 3. Frequency distribution of plant height (A) and peduncle length (B) of the MSD lines, their parent 'Norin 61' (triangle) and the adapted Sudanese cultivars 'Goumria' (black rhombus) and 'Imam' (white rhombus) of four environments of Dongola (DON), Wad Medani optimum sowing (MED/OS), Hudieba (HUD) and Wad Medani late sowing (MED/LS) in 2015/16.

$39 \%$ at HUD, and $38 \%$ at MED/LS did not head (Fig. 2). Among these lines, 16 were common between the cool environments DON and MED/OS, 110 between the hot environments HUD and MED/LS, and 16 between all environments.

The DH values were $62-104$ days at DON, 53-115 at MED/OS, 56-96 at HUD and 50-75 at MED/LS (Table 1). The mean DH value was lowest at MED/LS followed by HUD, MED/OS, and DON. This result reflected the differ- ences in temperature (cool at DON to hot at MED/LS; Fig. 1). We observed the same trend for DM. The DM values were lowest at MED/LS and highest at DON, whereas the GFD values were highest at HUD and lowest at MED/ LS (Table 1).

The variation in DH, DM, and GFD was greater in the MSD lines than in N61 and the two adapted Sudanese cultivars (Fig. 2, Supplemental Table 1). In comparison with $\mathrm{N} 61$, the DH values were significantly lower in $0.3 \%$ of the MSD lines at DON, $5 \%$ at MED/OS, $3 \%$ at HUD, and $4 \%$ at MED/LS, and were significantly higher in $40 \%$ of the MSD lines at DON, $36 \%$ at MED/OS, $55 \%$ at HUD, and $15 \%$ at MED/LS (Fig. 2A). In comparison with N61, the DM values were significantly lower in $1 \%$ of the MSD lines at DON and MED/OS, and in 5\% at HUD, and significantly higher in $35 \%$ of the MSD lines at DON, $27 \%$ at MED/OS, and $34 \%$ at HUD (Fig. 2B). In comparison with N61 and the two Sudanese cultivars, the GFD values were significantly higher in $5 \%$ of the MSD lines at MED/OS and in $11 \%$ at HUD, and significantly lower in $8 \%$ at MED/OS and in $19 \%$ at HUD (Fig. 2C).

The heritability of DH (0.87), DM (0.74), and GFD (0.76) was high across the four environments (Table 1). The genotype effect $(G)$ was significant for the three traits in all environments except for DM at MED/LS and GFD at DON and MED/LS (Supplemental Table 2). The environment effect $(E)$ and the $G \times E$ effect were significant for the three traits.

\section{Plant height and peduncle length}

The PH values were $47-136 \mathrm{~cm}$ at DON, $65-113$ at MED/OS, 55-110 at HUD, and 56-106 at MED/LS (Table 1). The mean PH values were highest at DON followed by MED/OS, MED/LS and HUD.

The variation in $\mathrm{PH}$ and PL was greater in the MSD lines

Table 1. Morphological, phenological, physiological, yield, and yield component traits of the MSD lines and their parent 'Norin 61' (N61) evaluated for heat stress tolerance at Dongola (DON), Hudeiba (HUD), Wad Medani with optimum sowing (MED/OS), and Wad Medani with late sowing (MED/LS) in Sudan

\begin{tabular}{|c|c|c|c|c|c|c|c|c|c|c|c|c|c|}
\hline \multirow{2}{*}{ Trait } & \multicolumn{3}{|c|}{ DON } & \multicolumn{3}{|c|}{ MED/OS } & \multicolumn{3}{|c|}{ HUD } & \multicolumn{3}{|c|}{ MED/LS } & \multirow{2}{*}{$\begin{array}{c}\text { Herita- } \\
\text { bility }\end{array}$} \\
\hline & Range & Mean & N61 & Range & Mean & N61 & Range & Mean & N61 & Range & Mean & N61 & \\
\hline $\mathrm{DH}$ & $62-104$ & 79 & 72 & $53-115$ & 72 & 64 & $56-96$ & 66 & 61 & $50-75$ & 63 & 62 & 0.87 \\
\hline $\mathrm{DM}$ & $99-135$ & 110 & 106 & $88-135$ & 108 & 101 & $94-125$ & 107 & 103 & $85-111$ & 97 & 92 & 0.74 \\
\hline GFD & $10-52$ & 32 & 33 & $11-61$ & 36 & 37 & $19-61$ & 40 & 42 & $20-45$ & 33 & 30 & 0.76 \\
\hline $\mathrm{PH}$ & $47-136$ & 102 & 97 & $65-113$ & 91 & 84 & $55-110$ & 80 & 69 & 56-106 & 84 & 79 & 0.71 \\
\hline PL & $20-53$ & 35 & 34 & $13-57$ & 32 & 82 & $8-32$ & 15 & 13 & 14-39 & 28 & 26 & 0.76 \\
\hline $\mathrm{CT}$ & $15-31$ & 23 & 32 & $13-31$ & 20 & 17 & $25-36$ & 30 & 30 & $21-37$ & 26 & 25 & 0.65 \\
\hline SPAD & $30-54$ & 44 & 43 & $27-68$ & 49 & 50 & $33-58$ & 51 & 51 & $38-60$ & 49 & 50 & 0.70 \\
\hline NDVI & $0.50-0.80$ & 0.68 & 0.71 & $0.27-0.84$ & 0.62 & 0.55 & $0.40-0.58$ & 0.65 & 0.63 & $0.54-0.89$ & 0.69 & 0.69 & 0.24 \\
\hline $\mathrm{KS}$ & $10-30$ & 20 & 23 & $10-65$ & 46 & 56 & $15-55$ & 41 & 45 & $10-65$ & 42 & 54 & 0.81 \\
\hline TKW & $15-55$ & 32 & 33 & $10-55$ & 30 & 30 & $15-40$ & 29 & 27 & $10-40$ & 27 & 26 & 0.88 \\
\hline $\mathrm{Gm}^{-2}$ & $2838-23268$ & 9142 & 10965 & $2250-48000$ & 21330 & 27181 & 2704-23874 & 9269 & 10357 & $2400-35200$ & 15726 & 21283 & 0.68 \\
\hline GY & $41-1172$ & 637 & 784 & $13-1053$ & 501 & 823 & $18-950$ & 421 & 383 & $8-891$ & 415 & 703 & 0.88 \\
\hline BIO & $500-4850$ & 2398 & 2366 & $300-3975$ & 2006 & 2038 & $99-2326$ & 1284 & 1279 & $310-3400$ & 1833 & 1089 & 0.60 \\
\hline $\mathrm{HI}$ & $2-63$ & 27 & 33 & $1-66$ & 25 & 40 & $6-60$ & 33 & 31 & $2-61$ & 23 & 39 & 0.89 \\
\hline
\end{tabular}

$\mathrm{DH}$, days to heading; DM, days to maturity; GFD, grain filling duration (days); PH, plant height (cm); PL, peduncle length (cm); CT, canopy temperature $\left({ }^{\circ} \mathrm{C}\right)$; SPAD, chlorophyll content (SPAD units); NDVI, normalized difference vegetation index; KS, kernel number per spike; TKW, thousand-kernel weight $(\mathrm{g})$; $\mathrm{Gm}^{-2}$, grain number per $\mathrm{m}^{2}$; $\mathrm{GY}$, grain yield $\left(\mathrm{g} \mathrm{m}^{-2}\right)$, BIO, biomass $\left(\mathrm{g} \mathrm{m}^{-2}\right)$; $\mathrm{HI}$, harvest index $(\%)$. 
than in N61 and the two adapted Sudanese cultivars. In comparison with $\mathrm{N} 61$, the $\mathrm{PH}$ values were significantly higher in $14 \%$ of the MSD lines at DON, $28 \%$ at MED/OS, $41 \%$ at $\mathrm{HUD}$, and $36 \%$ at MED/LS, and were significantly lower in $2 \%$ of the MSD lines at DON, $0.3 \%$ at MED/OS and $1 \%$ at MED/LS (Fig. 3A). In comparison with N61, the PL values were significantly higher in $8 \%$ of the MSD lines at DON, $29 \%$ at MED/OS, $18 \%$ at HUD, and $23 \%$ at MED/ LS, and were significantly lower in $5 \%$ of the MSD lines at DON, $2 \%$ at MED/OS, and $6 \%$ at MED/LS (Fig. 3B). The heritability of PH (0.71) and PL (0.76) was high (Table 1). The $G, E$, and $G \times E$ effects were significant for both traits (Supplemental Table 2).

\section{Canopy temperature, chlorophyll content, and normalized difference vegetation index}

The CT values were $15-31{ }^{\circ} \mathrm{C}$ at $\mathrm{DON}, 13-31^{\circ} \mathrm{C}$ at $\mathrm{MED} / \mathrm{OS}, 25-36^{\circ} \mathrm{C}$ at $\mathrm{HUD}$, and $21-37^{\circ} \mathrm{C}$ at $\mathrm{MED} / \mathrm{LS}$ (Table 1). The mean CT values were lowest at MED/OS followed by DON, MED/LS, and HUD. The SPAD values were $30-54$ at DON, 27-68 at MED/OS, 33-58 at HUD, and 38-60 at MED/LS (Table 1). The mean SPAD values were highest at HUD followed by MED/OS, MED/LS, and DON. The NDVI values were higher at MED/LS than at MED/OS. The variation in these three traits was higher in the MSD lines than in N61, 'Goumria', and 'Imam' (Table 1, Supplemental Table 1). In comparison with N61, the CT values were significantly lower in $10 \%$ of the MSD lines at HUD and were significantly higher in $23 \%$ at MED/ OS and in $8 \%$ at HUD (Fig. 4A). At HUD, the MSD lines that had lower CT values than N61 also had lower CT values than the Sudanese cultivars. In comparison with N61, the SPAD values were significantly higher in $7 \%$ of the MSD lines at DON, $3 \%$ at MED/OS, and $2 \%$ at MED/LS, and were significantly lower in $3 \%$ of the MSD lines at DON, $10 \%$ at MED/LS, and $7 \%$ at MED/OS and HUD (Fig. 4B). In comparison with N61, the NDVI values were significantly higher in $30 \%$ of the MSD lines at MED/OS and in $6 \%$ at MED/LS, and were significantly lower in $5 \%$ of the MSD lines at MED/OS and MED/LS (Fig. 4C). Comparing to the adapted Sudanese cultivars, the MSD lines that had cooler canopy than N61 also had cooler canopy than the adapted Sudanese cultivars at HUD. The heritability of SPAD (0.65) and CT (0.70) was high, whereas the heritability of NDVI (0.24) was low (Table 1). The G effect was significant for SPAD in all environments, for CT at MED/OS and HUD, and for NDVI at MED/OS and MED/ LS (Supplemental Table 2). The $\mathrm{E}$ and $\mathrm{G} \times \mathrm{E}$ effects were significant for the three traits.

Kernel number per spike, thousand-kernel weight, and grain number per $\mathrm{m}^{2}$

The KS values were 10-30 at DON, 10-65 at MED/OS, 15-55 at HUD, and 10-65 at MED/LS (Table 1). The mean
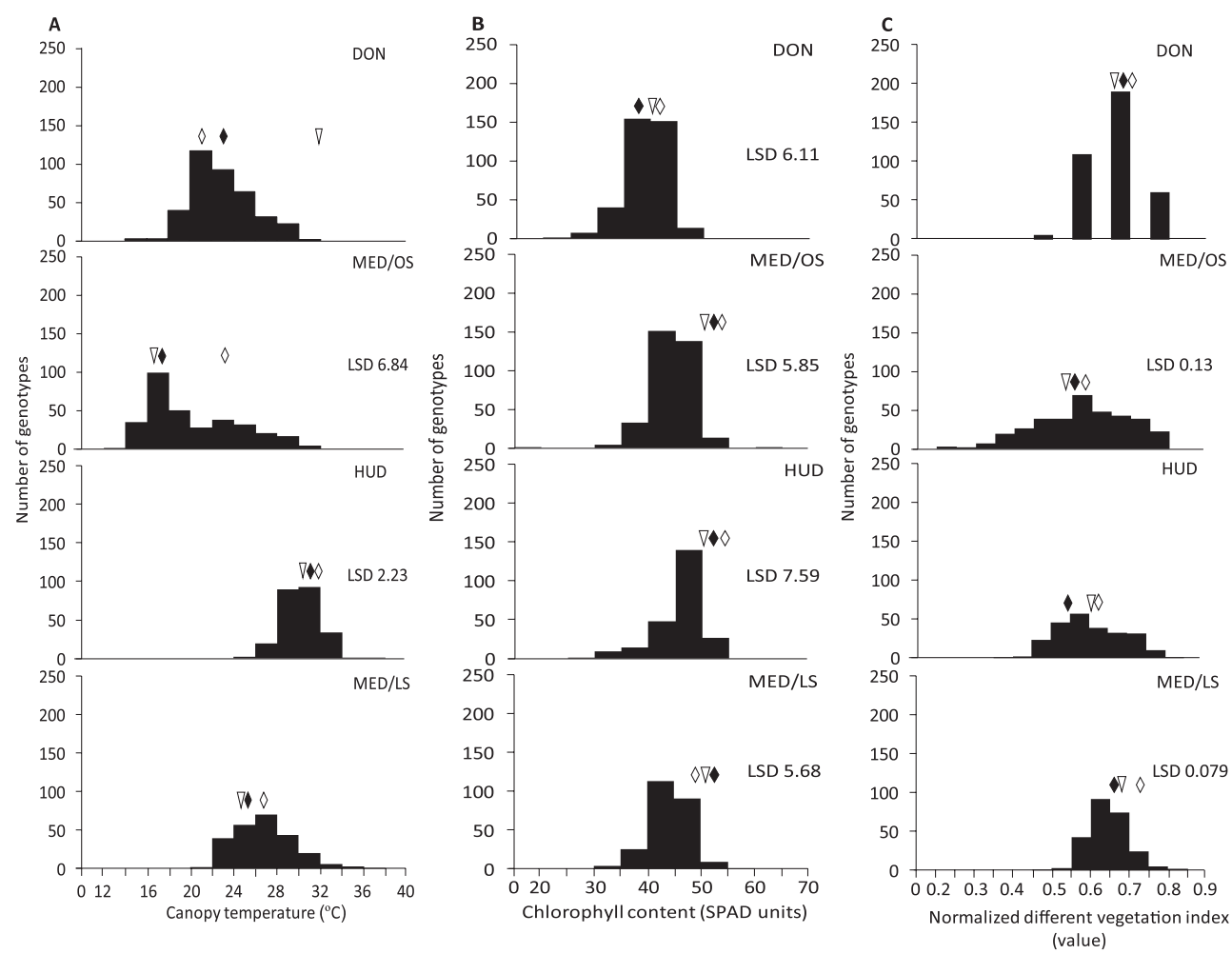

Fig. 4. Frequency distribution of canopy temperature (A) chlorophyll content (B) and normalized difference vegetation index (C) of the MSD lines, their parent 'Norin 61' (triangle) and the adapted Sudanese cultivars 'Goumria' (black rhombus) and 'Imam' (white rhombus) of four environments of Dongola (DON), Wad Medani optimum sowing (MED/OS), Hudieba (HUD) and Wad Medani late sowing (MED/LS) in 2015/16. 
$\mathrm{KS}$ value was highest at MED/OS followed by those at MED/LS, HUD, and DON. In comparison with N61, KS values were higher in $2 \%$ of the MSD lines at DON and significantly lower in 14\% (DON), 27\% (MED/OS), 5\% (HUD), and 26\% (MED/LS) (Fig. 5A).

The TKW values were 15-55 g at DON, 10-55 at MED/ OS, 15-40 at HUD, and 10-40 at MED/LS (Table 1). The mean TKW value was highest at DON followed by MED/ OS, HUD and MED/LS. In comparison with N61, TKW values were significantly higher in $6 \%$ (DON) and $15 \%$ (HUD) of the MSD lines, and were significantly lower in 16\% (DON) and 6\% (HUD) (Fig. 5B). These results indicate the impact of the genes from the different synthetic wheat in these lines. The MSD lines that were higher in TKW than N61 were also higher than the adapted Sudanese cultivars.

The $\mathrm{Gm}^{-2}$ values were 2838-23268 at DON, 2250 48000 at MED/OS, 2704-23874 at HUD, and 2400-35200 at MED/LS (Table 1). The mean $\mathrm{Gm}^{-2}$ value was highest at MED/OS, followed by those at MED/LS, HUD, and DON. In comparison with $\mathrm{N} 61, \mathrm{Gm}^{-2}$ values were significantly higher in 3\% (MED/OS), 6\% (HUD), and 5\% (MED/LS) of the MSD lines, and were significantly lower in $42 \%$ (MED/ OS), 12\% (HUD), and 25\% (MED/LS) (Fig. 5C). The MSD lines that had higher values of all three traits than N61 also had higher values than the Sudanese cultivars. The heritability of all three traits was high: 0.81 for KS, 0.88 for TKW, and 0.68 for $\mathrm{Gm}^{-2}$ (Table 1).

The $\mathrm{G}$ effect was significant for the three traits in all environments except for TKW at MED/OS and MED/LS and $\mathrm{Gm}^{-2}$ at DON (Supplemental Table 2). The E and $\mathrm{G} \times \mathrm{E}$ effects were significant for the three traits.

\section{Grain yield, biomass, and harvest index}

The GY values were $41-1172 \mathrm{~g} \mathrm{~m}^{-2}$ at DON, 13-1053 $\mathrm{g} \mathrm{m}^{-2}$ at MED/OS, 18-950 $\mathrm{g} \mathrm{m}^{-2}$ at HUD, and 8-891 $\mathrm{g} \mathrm{m}^{-2}$ at MED/LS (Table 1). The mean GY value was highest at DON followed by MED/OS, HUD, and MED/LS. This result was consistent with the low temperature at DON and high temperature at MED/LS (Fig. 1). The mean BIO value was greatest at DON and lowest at HUD. The mean HI value was highest at HUD followed by DON, MED/OS and MED/LS (Table 1). The lowest BIO at HUD and lowest HI at $\mathrm{MED} / \mathrm{LS}$ can be attributed to their high temperatures compared to those of DON and MED/OS (Table 1, Fig. 1).

In comparison with N61, GY values were significantly higher in $10 \%$ of the MSD lines (28 lines) at HUD, and were significantly lower in $38 \%$ at $\mathrm{MED} / \mathrm{OS}$ and $7 \%$ at HUD (Fig. 6A). In comparison with N61, BIO values were significantly higher in 3\% of the MSD lines at MED/OS and $10 \%$ at HUD, and were significantly lower in $4 \%$ at MED/ OS and $8 \%$ at HUD (Fig. 6B). In comparison with N61, HI values were significantly lower in $0.6 \%$ of the MSD lines at $\mathrm{MED} / \mathrm{OS}$ and in 5\% at HUD (Fig. 6C).
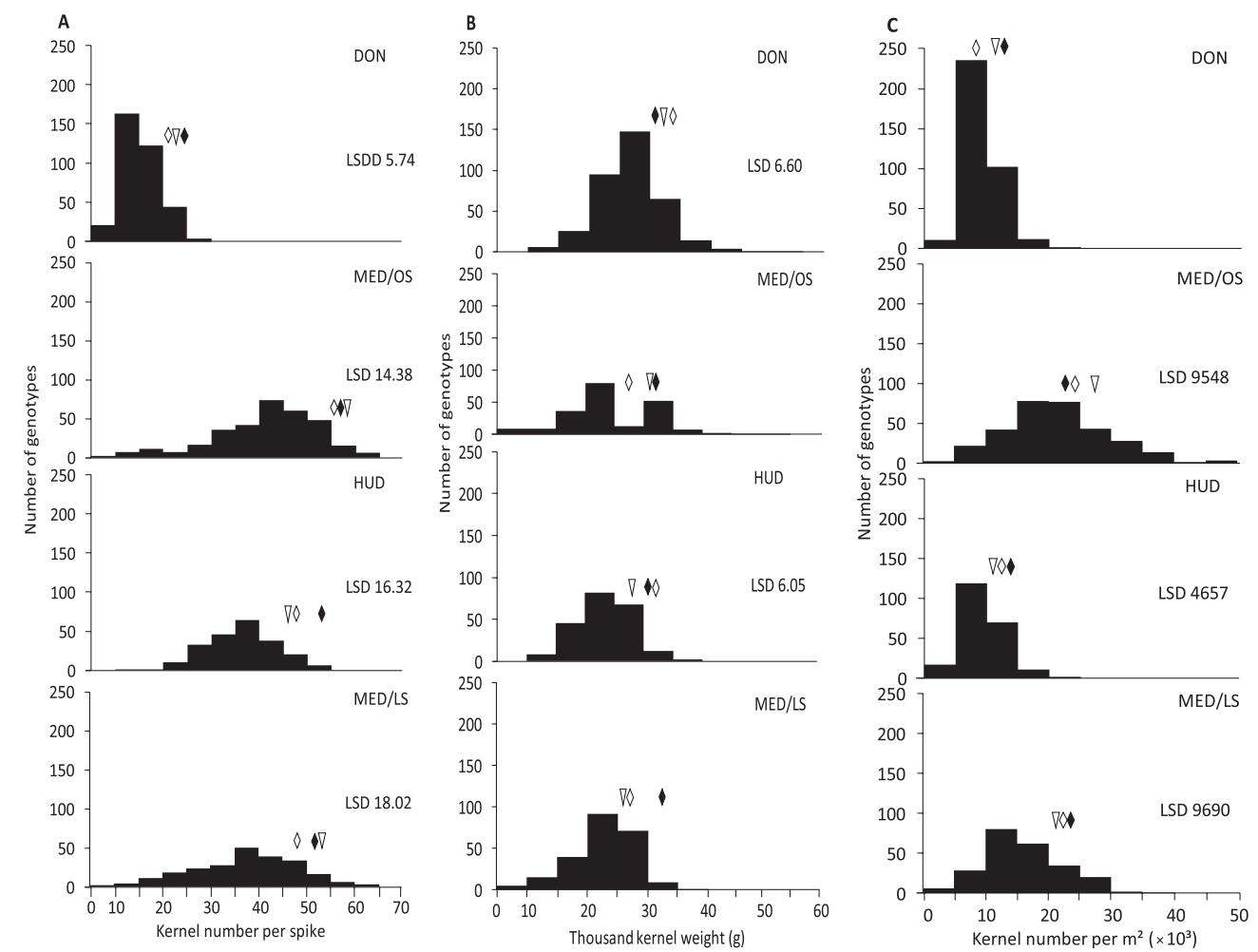

Fig. 5. Frequency distribution of kernel number per spike (A), thousand-kernel weight (B) and grain number per $\mathrm{m}^{2}(\mathrm{C})$ of the MSD lines, their parent 'Norin 61' (triangle) and the adapted Sudanese cultivars 'Goumria' (black rhombus) and 'Imam' (white rhombus) of four environments of Dongola (DON), Wad Medani optimum sowing (MED/OS), Hudieba (HUD) and Wad Medani late sowing (MED/LS) in 2015/16. 

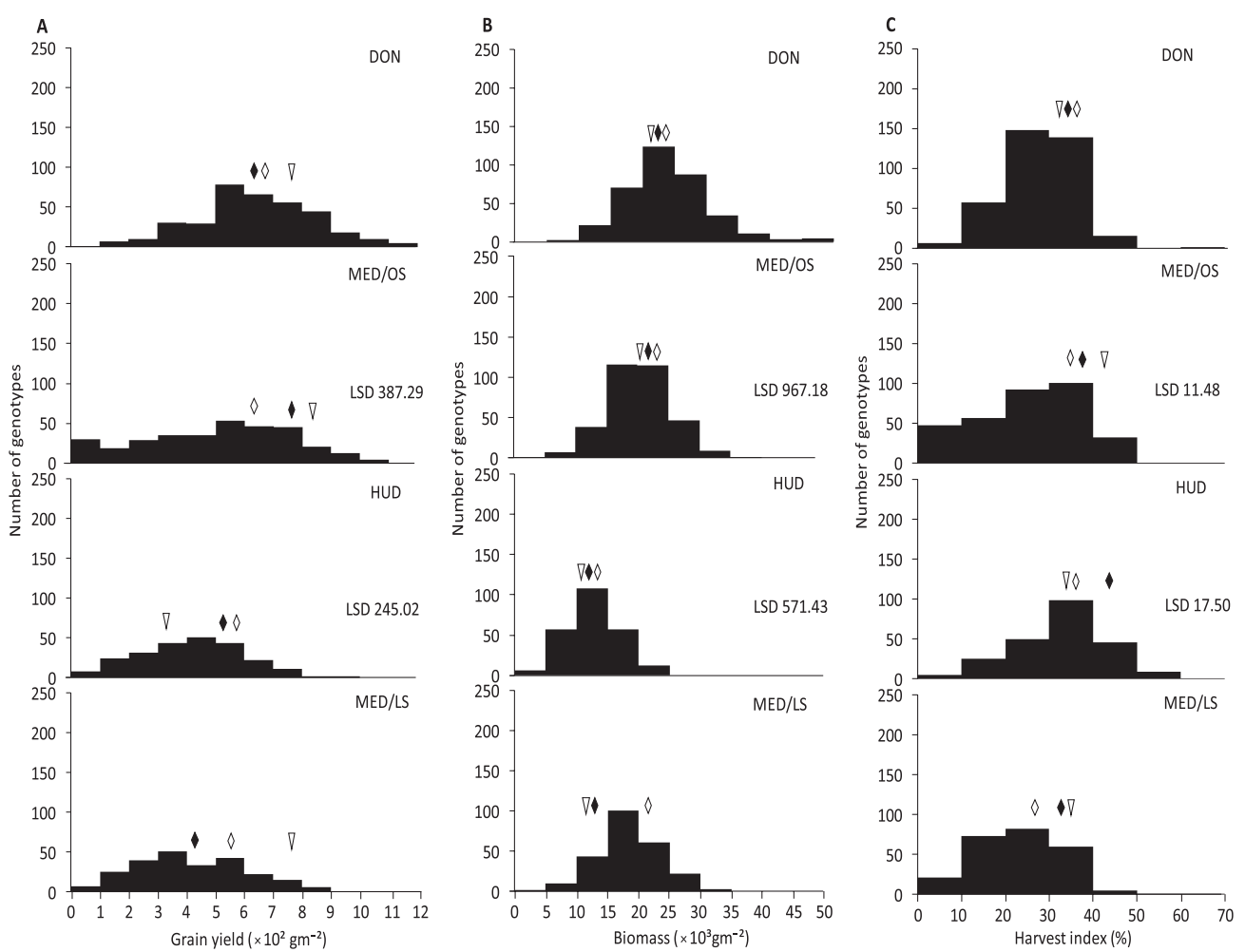

Fig. 6. Frequency distribution of grain yield (A), biomass (B) and harvest index (C) of the MSD lines, their parent 'Norin 61' (triangle) and the adapted Sudanese cultivars 'Goumria' (black rhombus) and 'Imam' (white rhombus) of four environments of Dongola (DON), Wad Medani optimum sowing (MED/OS), Hudieba (HUD) and Wad Medani late sowing (MED/LS) in 2015/16.

In comparison with 'Goumria' and 'Imam', GY values were significantly higher in 3\% of the MSD lines at HUD; BIO values were significantly higher in $3 \%$ of the MSD lines at MED/OS and in 4\% at HUD; and HI values were significantly higher in $2 \%$ of the MSD lines at MED/OS and in $3 \%$ at HUD. The heritability of GY (0.88) and HI (0.89) was high (Table 1) and that of BIO (0.60) was moderate. The $\mathrm{G}$ effect was significant for the three traits at HUD and MED/OS (Supplemental Table 2). The E and G $\times$ E effects were significant for the three traits.

\section{Heat tolerance efficiency}

The HTE values calculated from GY at DON and MED/ LS were 14-151\% (first calculation; N61, 90\%) and 15$173 \%$ when calculated from GY at MED/OS and MED/LS (second calculation; N61, 85\%). In comparison with N61, HTE was higher in 38 MSD lines (17\%) in the first calculation (range, 93-151\%; Fig. 7A) and in 56 MSD lines (24\%) in the second calculation (range, $88-173 \%$; Fig. 7B). We considered the lines with HTE above $100 \%$ as highly heat tolerant and found 13 lines (Supplemental Table 3) consistent between both calculations.

To classify the MSD lines according to their GY and HTE, we performed a regression analysis of the relationship between HTE from the first calculation and GY at DON (cool environment), and between HTE from the second calculation and GY at MED/OS (cool environment). In both analyses, no MSD lines showed higher GY with HTE than those of N61 (Fig. 7). Higher HTE values and lower yield than N61 were found in $17 \%$ of the MSD lines in the first analysis (Fig. 7A) and in $24 \%$ in the second analysis (Fig. 7B). These MSD lines also had higher HTE than the Sudanese cultivars in both analyses.

\section{Association of grain yield with morphological, phenologi- cal, and physiological traits}

The correlation of GY with some heat tolerance-related traits among the MSD lines is shown in Table 2. GY was negatively correlated with DH in all environments. GY was negatively correlated with DM and positively correlated with GFD in all environments except for MED/LS, where correlations between GY and these two traits were not significant. PL was positively correlated with GY at MED/OS and MED/LS, was negatively correlated at DON, and did not correlate with GY at HUD.

$\mathrm{BIO}, \mathrm{HI}, \mathrm{KS}, \mathrm{TKW}$, and $\mathrm{Gm}^{-2}$ were positively correlated with GY in all environments, except that TKW did not correlate with GY at DON. At MED/OS, CT was negatively correlated with GY, whereas SPAD was positively correlated. NDVI was positively correlated with GY at MED/OS and weakly negatively correlated at MED/LS. HTE showed a negative correlation with GY of DON and MED/OS. 

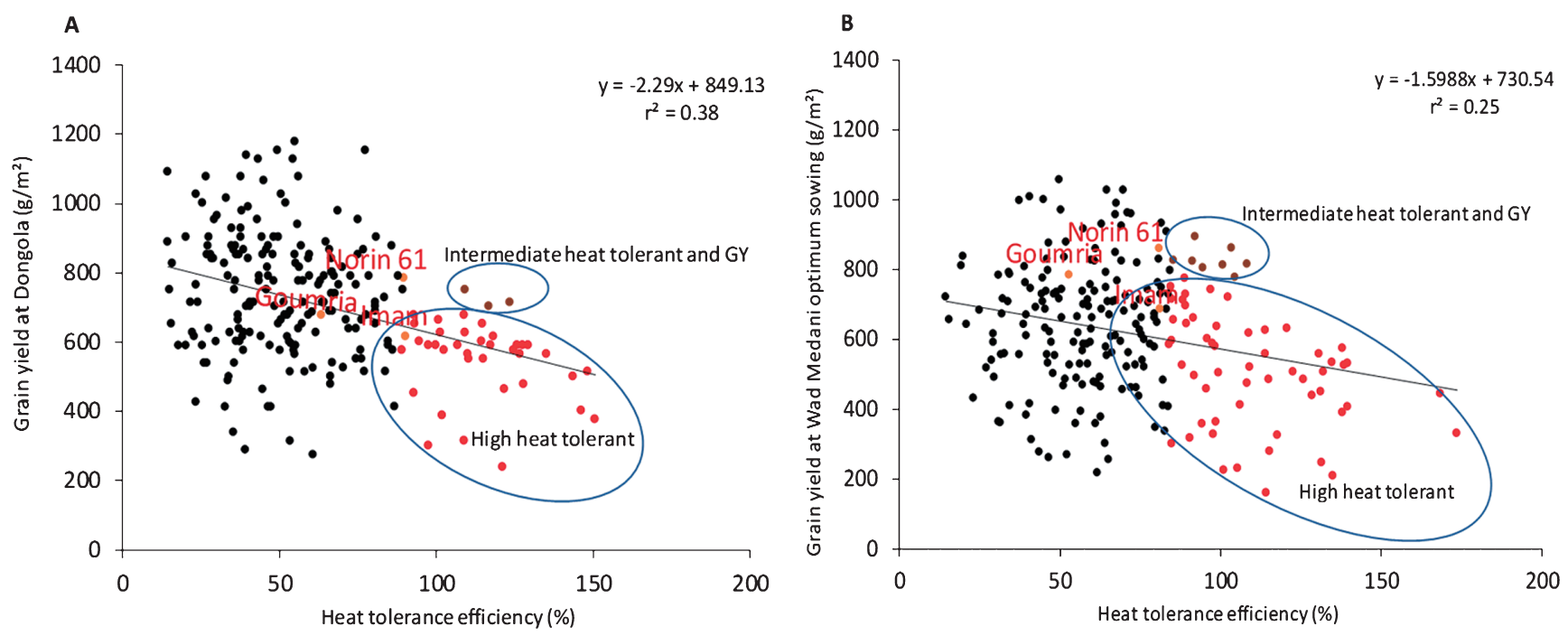

Fig. 7. Regression analysis of the relationship between grain yield at DON and heat tolerance efficiency DON (considered a cool environment) and at MED/LS (hot environment) (A) and relationship between grain yield at MED/OS and heat toleance efficiency MED/OS (considered a cool environment) and at MED/LS (considered a hot environment) (B) for the MSD lines, their parent 'Norin 61' (control), and the adapted Sudanese cultivars 'Goumria' and 'Imam'. Red dots, highly heat-tolerant lines; dark orange dots, moderately heat-tolerant lines with good grain yield.

Table 2. Correlation coefficients between grain yield and the phenological, morphological, physiological, and yield component traits of the MSD lines at Wad Medani with optimum sowing (MED/OS), Wad Medani with late sowing (MED/LS), Dongola (DON), and Hudeiba (HUD)

\begin{tabular}{lcccc}
\hline \hline Trait & MED/OS & MED/LS & DON & HUD \\
\hline DH & $-0.52^{* * *}$ & $-0.33^{* * *}$ & $-0.17^{* * *}$ & $-0.29 * * *$ \\
DM & $-0.50^{* *}$ & $-0.10^{\text {ns }}$ & $-0.15^{* * *}$ & $-0.22^{* * *}$ \\
GFD & $0.27^{* * *}$ & $-0.01^{\text {ns }}$ & $0.17^{* *}$ & $0.13^{*}$ \\
PH & $0.26^{* * *}$ & $0.04^{\text {ns }}$ & $-0.17^{* *}$ & $-0.09^{\text {ns }}$ \\
PL & $0.32 * * *$ & $0.13^{*}$ & $-0.13^{*}$ & $-0.03^{\text {ns }}$ \\
CT & $-0.11^{*}$ & $-0.07^{\text {ns }}$ & $0.006^{\text {ns }}$ & $-0.10^{\text {ns }}$ \\
SPAD & $0.14 *$ & $0.11^{\text {ns }}$ & $-0.05^{\text {ns }}$ & $0.03^{\text {ns }}$ \\
NDVI & $0.39 * * *$ & $-0.18^{* *}$ & $0.02^{\text {ns }}$ & $0.03^{\text {ns }}$ \\
KS & $0.41^{* * *}$ & $0.21^{*}$ & $0.21^{* * *}$ & $0.28^{* * *}$ \\
TKW & $0.41^{* * *}$ & $0.26^{* * *}$ & $0.09^{\text {ns }}$ & $0.39 * * *$ \\
Gm ${ }^{-2}$ & $0.31 * * *$ & $0.39^{* * *}$ & $0.33^{* * *}$ & $0.54 * * *$ \\
BIO & $0.42^{* * *}$ & $0.38^{* * *}$ & $0.47^{* * *}$ & $0.59 * * *$ \\
HI & $0.81 * * *$ & $0.74^{* * *}$ & $0.60^{* * *}$ & $0.52^{* * *}$ \\
HTE & $-0.25^{* * *}$ & & $-0.54^{* * *}$ & \\
\hline
\end{tabular}

$*, * *, * * *$ significant at the $0.05,0.01,0.001$ probability levels, respectively; ns, non-significant; DH, days to heading; DM, days to maturity; GFD, grain filling duration; $\mathrm{PH}$, plant height; PL, peduncle length, CT, canopy temperature; SPAD, chlorophyll content; NDVI, normalized difference vegetation index; KS, kernel number per spike; TKW, thousand-kernel weight; $\mathrm{Gm}^{-2}$, grain number per $\mathrm{m}^{2}$; BIO, biomass; HI, harvest index; HTE, heat tolerance efficiency.

\section{Discussion}

In this study, we used a unique population of diverse lines derived from $43 \mathrm{Ae}$. tauschii accessions in a hexaploid wheat background (N61). We evaluated this population in four different environments with N61 and two adapted Sudanese cultivars, and found considerable genetic variation in all traits examined (Table 1). All traits except NDVI had moderate to high heritability, suggesting that the selection for all these traits within the MSD population will be effective.

The responses of DH and DM depended on the differences in temperature and environments (Table 1, Fig. 2A, 2B); the average DH values were higher in the cool environments (DON and MED/OS) than in the hot environments (HUD and MED/LS) (Table 1). The GFD values were higher in some lines than in N61 and the Sudanese cultivars, and lower in some other lines (Table 1, Supplemental Table 1, Fig. 2C). These results indicate the ability of some MSD lines to adapt to heat stress by shortening the GFD depending on the degree of temperature. In each environment, one group of lines headed and matured earlier than N61, another group headed and matured later than N61, and the third group was intermediate. This grouping is very important for wheat breeding under heat stress because of the temperature fluctuations during the crop cycle. These lines might also be suitable for drought tolerance breeding because of the fluctuation of the timing of rain between different locations and years. The variation in DH allows wheat genotypes to optimize their use of resources (Laurie 1997). Early-maturing genotypes are preferable for Sudanese environments, where grain yield is limited by high temperatures and the wheat cycle is short (Lopes et al. 2012, Reynolds et al. 1994). Most of the MSD lines headed later than N61 and some lines were unable to head (winter growth habit) in any of the four environments (Table 1, Fig. 2A). Most of the 43 SHW parental lines did not head at MED. Thus, we attributed late heading and the winter growth habit of the MSD lines to the vernalization requirement inherited from their SHW parents (our unpublished data). The number of the MSD lines that did not flower was highest at HUD and MED/LS, whereas 
the number of the MSD lines that headed was highest in MED/OS, likely because the minimum temperatures from the mid-vegetative growth stage to the beginning of heading (middle of the $6^{\text {th }}$ week- $8^{\text {th }}$ week) at MED/OS were lower than those at HUD and MED/LS at late vegetative growth and beginning of heading. The low night temperature at MED/OS might be suitable for wheat to become flowering competent.

Blum (1998) reported that PL plays a role in the storage of water-soluble carbohydrates under stress when GFD is short. Therefore, longer peduncles are preferable under heat stress to support grain filling. In our study, PL was correlated with GY (Table 2). The MSD lines exhibited wide variation in PL (Table 1), and PL values were higher in some lines than in N61 and the Sudanese cultivars. These lines are promising in programs aimed to improve GY under heat stress.

$\mathrm{Gm}^{-2}$ greatly contributes to high yield in heat stress environments (Fischer 2008). Selection for BIO and HI is an efficient method for selecting high-yielding genotypes under heat stress (He and Rajaram 1993, Tahir and Nakata 2005). In our study, KS, TKW, $\mathrm{Gm}^{-2}$, BIO, and HI were correlated with GY, and their values were higher in a number of MSD lines than in N61 and the Sudanese cultivars in each environment (Figs. 5, 6). These lines could serve as an excellent resource to improve these traits and hence GY under heat stress conditions.

CT, SPAD, and NDVI varied widely among the MSD lines, and their values were better in some lines than in N61 (Fig. 4). Low CT, high SPAD, and high NDVI are reliable indicators of heat-tolerant and high-yielding genotypes in wheat (Lopes et al. 2012, Olivares-Villegas et al. 2007, Shahnoza et al. 2012). However, in this study, we found a weak correlation between these traits and GY (Table 2). These traits are highly affected by the phenology and $\mathrm{PH}$ (Lopes et al. 2012). Our population exhibited wide variation in heading and maturity (Table 1), and therefore we attribute the weak correlation to the inconsistency in the time of measurements for different plants.

We found 29 MSD lines with significantly higher GY than that of N61 at HUD and a number of other MSD lines that tended to have higher GY than N61, indicating a high frequency of favorable alleles for GY in the population. These lines can be used in breeding programs to increase yield potential without major concerns about the presence of alleles with negative effects. The 29 high-yielding MSD lines responded differently to heat stress, and each of them had different characteristics that increased productivity (Supplemental Table 4). For example, line MSD215 headed early, and some other lines had lower CT or higher PL, $\mathrm{KS}, \mathrm{TKW}, \mathrm{Gm}^{-2}$, BIO, or HI values.

Using HTE, we were able to estimate the heat tolerance of each line. In 13 lines, HTE values were consistently higher than in N61 (Supplemental Table 3). The negative correlation between GY and HTE indicates that the heat tolerance is associated with lower yielding ability, which is expected in this $\mathrm{BC}_{1}$ population, as the lines are not expected to perform as well as N61. The HTE of some lines was intermediate between those of N61 and highly tolerant lines with good yield potential (Fig. 7); these lines are a good source to improve both GY and heat tolerance. The characteristics of highly tolerant lines were similar to those of N61, but with low GY (Supplemental Table 3). More detailed physiological analysis is needed to explain the heat tolerance of these lines. In comparison with N61, some of these lines showed insignificantly lower CT and higher PL, $\mathrm{KS}, \mathrm{TKW}$, and BIO values (Supplemental Table 3). Some of them had significantly higher CT than N61, indicating that their mechanism of tolerance differs from $\mathrm{CT}$ reduction through high transpiration, which would make these lines suitable for cultivation under drought. The pedigree of these lines (Supplemental Table 4) indicated that 9 of the 43 SHW lines contributed to the tolerance.

GY could be boosted by combining traits conferring agronomic and physiological tolerance under heat stress in the same cultivar. Reynolds et al. (2009) reported that "strategic trait-based crossing" results in progeny that has superior yield and yield-related physiological traits in comparison with conventionally bred advanced lines. Strategic traitbased crossing requires characterization of large sets of germplasm to find lines with desirable traits; such germplasm can be crossed to introduce positive alleles for each trait into new genetic backgrounds (Lopes et al. 2012).

In this study, we used 400 synthetic wheat lines and identified several lines with traits significantly associated with adaptation to heat stress. These lines are an excellent source of alleles to improve wheat adaptation and tolerance to heat stress through the strategic trait-based crossing. In this study, only a small number of lines excelled in several traits studied in comparison with N61. These results indicate that our approach is very effective and could be the best way to explore and use the variation in heat tolerance in Ae. tauschii in a practical, fast, and economical way. We are performing genomic analysis of these 400 MSD lines to conduct a genome-wide association study and identify the quantitative trait loci (QTLs) associated with each trait; this information would facilitate the introgression of these QTLs through marker-assisted selection.

\section{Acknowledgments}

This work was partly supported by JSPS KAKENHI (No. 15H04037) and the MRA Project of Tottori University.

\section{Literature Cited}

Al-Khatib, K. and G.M. Paulsen (1990) Photosynthesis and productivity during high-temperature stress of wheat genotypes from major world regions. Crop Sci. 30: 1127-1132.

Allard, R.W. (1960) Principles of plant breeding, John Wiley and Sons, New York, USA.

Amani, I., R.A. Fischer and M.P. Reynolds (1996) Canopy temperature depression association with yield of irrigated spring wheat cultivars in a hot climate. J. Agric. Crop Sci. 176: 119-129. 
Asseng, S., I. Foster and N.C. Turner (2011) The impact of temperature variability on wheat yields. Glob. Change Biol. 17: 997-1012.

Asseng, S., F. Ewert, P. Martre, R.P. Rötter, D.B. Lobell, D. Cammarano, B.A.Kimball, M.J.Ottman, G.W.Wall, J.W.White et al. (2015) Rising temperatures reduce global wheat production. Nat. Clim. Chang. 5: 143-147.

Blum,A. (1998) Improving wheat grain filling under stress by stem reserve mobilisation. Euphytica 100: 77-83.

Elbashir,A.A.E., Y.S.A. Gorafi, I.S.A. Tahir, J.-S. Kim and H. Tsujimoto (2017) Wheat multiple synthetic derivatives: a new source for heat stress tolerance adaptive traits. Breed. Sci. 67: 248-256.

Ferris, R., R.H.Ellis, T.R. Wheeler and P.Hadley (1998) Effect of high temperature stress at anthesis on grain yield and biomass of fieldgrown crops of wheat. Ann. Bot. 82: 631-639.

Fischer, K.S. and G. Wood (1981) Breeding and selection for drought tolerance in tropical maize. In: Fischer,K.S., E.S.Jonson and G.O.Edmeades (eds.) Principles and Methods in Crop Improvement for Drought Resistance with Emphasis on Rice. Proceeding of the Symposium. IRRI, Philippines, pp. 31-46.

Fischer, R.A. (2008) The importance of grain or kernel number in wheat: a reply to Sinclair and Jamieson. Field Crops Res. 105: 1521.

Hazratkulova, S., R. Sharma, S.Alikulov, S. Islomov, T. Yuldashev, Z. Ziyaev, Z. Khalikulov, Z.Ziyadullaev and J. Turok (2012) Analysis of genotypic variation for normalized difference vegetation index and its relationship with grain yield in winter wheat under terminal heat stress. Plant Breed. 131: 716-721.

He,Z. and S. Rajaram (1993) Differential responses of bread wheat characters to high temperature. Euphytica 72: 197-203.

Kazi, M. and M. Van Ginkel (2004) Wild wheat relatives help boost genetic diversity. CIMMYT News.

Laurie, D.A. (1997) Comparative genetics of flowering time. Plant Mol. Biol. 35: 167-177.

Li, J., H.-S. Wan and W.-Y. Yang (2014) Synthetic hexaploid wheat enhances variation and adaptive evolution of bread wheat in breeding processes. J. Syst. Evol. 52: 735-742.

Lobell, D.B., A. Sibley and J.I. Ortiz-Monasterio (2012) Extreme heat effects $n$ wheat senescence in India. Nat. Clim. Chang. 2: 186-189.

Lopes, M.S., M.P. Reynolds, M.R. Jalal-Kamali, M. Moussa, Y.Feltaous, I.S.A. Tahir, N. Barma, M. Vargas, Y. Mannes and M. Baum (2012) The yield correlations of selectable physiological traits in a population of advanced spring wheat lines grown in warm and drought environments. Field Crops Res. 128: 129-136.

Matsuoka, Y. and S. Nasuda (2004) Durum wheat as a candidate for the unknown female progenitor of bread wheat: an empirical study with a highly fertile $\mathrm{F}_{1}$ hybrid with Aegilops tauschii Coss. Theor. Appl. Genet. 109: 1710-1717.

Olivares-Villegas, J.J., M.P.Reynolds and G.K.McDonald (2007) Drought-adaptive attributes in the Seri/Babax hexaploid wheat population. Funct. Plant Biol. 34: 189-203.

Pask,A.J.D., J.Pietragalla, D.M.Mullan and M.P.Reynolds (eds.) (2012) Physiological Breeding II: A field guide to wheat phenotyping. Mexico, D.F.: CIMMYT.

Reynolds, M.P., M. Balota, M.I.B. Delgado, I.Amani and R.A. Fischer (1994) Physiological and morphological traits associated with spring wheat yield under hot, irrigated conditions. Aust. J. Plant Physiol. 21: 717-730.

Reynolds, M.P., Y. Manes, A. Izanloo and P.Langridge (2009) Phenotyping for physiological and morphological breeding and gene discovery in wheat. Ann. Appl. Bio1. 155: 309-320.

Shahnoza,H., R.C.Sharma, S.Alikulov, S. Islomov, T. Yuldashev, Z. Ziyaev, Z. Khalikulov, Z. Ziyadullaev and J. Turok (2012) Analysis of genotypic variation for normalized difference vegetation index and its relationship with grain yield in winter wheat under terminal heat stress. Plant Breed. 131: 716-721.

Sharma, P., S. Sareen, M.Saini, A. Verma, B.S. Tyagi and I. Sharma (2014) Assessing genetic variation for heat tolerance in synthetic wheat lines using phenotypic data and molecular markers. Aust. J. Crop Sci. 8: 515-522.

Tahir, I.S.A. and N. Nakata (2005) Remobilization of nitrogen and carbohydrate from stems of bread wheat in response to heat stress during grain filling. J. Agron. Crop Sci. 191: 105-115.

Tahir,I.S.A., N. Nakata, A.M.Ali, H.M. Mustafa, A.S.I.Saad, K. Takata, N. Ishikawa and O.S.Abdalla (2006) Genotypic and temperature effects on wheat grain yield and quality in a hot irrigated environment. Plant Breed. 125: 323-330.

Tewolde, H., C.J.Fernandez and C.A. Erickson (2006) Wheat cultivars adapted to post-heading high temperature stress. J. Agron. Crop Sci. 192: 111-120.

Tsujimoto,H., Q. Sohail and Y.Matusoka (2015) Broadening the genetic diversity of common and durum wheat for abiotic stress tolerance breeding. In: Ogihara,Y., S.Takumi and H.Handa (eds.) Advances in Wheat Genetics: From Genome to Field. Proceedings of the $12^{\text {th }}$ International Wheat Genetics Symposium. Yokohama, Japan, Springer, pp. 233-238.

Van Ginkel, M. and F. Ogbonnaya (2007) Novel genetic diversity from synthetic wheats in breeding cultivars for changing production conditions. Field Crops Res. 104: 86-94. 